\title{
FREE ACTIONS AND COMPLEX COBORDISM
}

\author{
CONNOR LAZAROV AND ARTHUR G. WASSERMAN ${ }^{1}$
}

ABSTRACT. Connor and Floyd have observed that a free action of a finite group $G$ on a compact manifold $M$ preserving a stable almost complex structure produces a stably almost complex quotient manifold $M / G$. Hence, the bordism group of such actions, $U_{*}$, free, is just $U_{*}(B G)$. If $G$ is not finite or abelian, but an arbitrary compact Lie group, the tangent bundle along the fibres gives trouble. Nevertheless, it is shown that if $H^{*}(B G)$ is torsion free then $U_{*}$, free $\approx U_{*}(B G)$.

In [2] it was shown that the bordism group of free actions of a compact Lie group $G$ on stably almost complex manifolds (preserving the complex structure) is a free $M U_{*}$ module if $G$ is connected and $H^{*}(G)$ is torsion free. The proof involved a detailed computation of the cohomology of the spectrum associated to the bordism theory as a module over the Steenrod algebra and as an $H^{*}(M U)$ comodule. In the present paper, a geometric approach yields the same result much more simply. We would like to thank R. Stong for a helpful conversation.

Let $X$ be a topological space and $E$ a $k$-plane bundle on $X$. Let $\Omega_{n}^{U}(X, E)$ be the bordism group of triples $(M, f, J)$ where $M^{n-k}$ is a closed manifold, $f: M \rightarrow X$ is continuous and $J$ is a stable complex structure on $T(M) \oplus f^{*} E .(M, f, J)$ is bordant to $\left(M^{\prime}, f^{\prime}, J^{\prime}\right)$ if there is a $\left(W^{n-k+1}, F, J^{\prime \prime}\right)$ with $\partial W=M \cup M^{\prime}, F|M=f, F| M^{\prime}=f^{\prime}, J^{\prime \prime} \mid M=J$ and $J^{\prime \prime} \mid M^{\prime}=-J^{\prime} . \Omega_{*}^{U}(X, E)$ is a $U_{*}$ module by $\left(N, J^{\prime}\right) \cdot(M, f, J)=\left(N \times M, J^{\prime} \times J, f \circ p\right)$, where $p$ : $N \times M \rightarrow M$ is the projection.

Proposition 1. $\Omega_{*}^{U}(X, E)=U_{*}(D(E), S(E))$ as $U_{*}$ modules.

Proof. Let $\theta: \Omega_{*}^{U}(X, E) \rightarrow U_{*}(D(E), S(E))$ be defined by $\theta(M ; f ; J)=$ $\left(D\left(f^{*} E\right) ; \bar{f} ; \pi^{*} J\right)$, where $\bar{f}$ is the map from $f^{*} E$ to $E$ induced by $f$, and $D$ (resp. $S$ ) denotes the disc (resp. sphere) bundle. Note that the tangent bun-

Received by the editors November 16, 1973.

AMS (MOS) subject classifications (1970). Primary 57D85, 57D90; Secondary 57E 15.

Key words and phrases. Free actions, complex bordism.

1 This research was supported in part by NSF grants GP-12639 and GP 7952X3. 
dle of $f^{*} E, T\left(f^{*} E\right)$, is $\pi^{*} T\left(f^{*} E\right) \mid M=\pi^{*}\left(T(M) \oplus f^{*} E\right)$, where $\pi: f^{*} E \rightarrow M$, and hence the stable complex structure $J$ induces a stable complex structure $\pi^{*} J$ on $f^{*} E$. Clearly, $\theta$ is a well-defined $U_{*}$ homomorphism.

Let $\phi: U_{*}(D(E), S(E)) \rightarrow \Omega_{*}^{U}(X, E)$ be defined as follows: an element $x \in U_{*}(D(E), S(E))$ is represented by a map $F:(Q, \partial Q) \rightarrow(D(E), S(E))$ and a stable complex structure $J$ on $Q$; we may choose $F$ to be "transverse regular" to the zero section of $E$ so that $M=F^{-1}(X) \subset Q$ is a closed submanifold. Let $F \mid M=f$; then the normal bundle of $M$ in $Q, \nu(M, Q)=$ $f^{*} \nu(X, E)=f^{*} E$. Also $T(Q) \mid M=T(M) \oplus \nu(Q, M)=T(M) \oplus f^{*} E$; hence the restriction of $J$ to $T(Q)|M, J| M$, is a stable complex structure on $T(M) \oplus f^{*} E$. We set $\phi(x)=(M, f, J \mid M)$. The usual arguments show that $\phi$ is a well-defined $U_{*}$ homomorphism. Clearly, $\phi \circ \theta=$ identity on $\Omega_{*}^{U}(X, E)$, and we need only show that $\theta \circ \phi=$ identity on $U_{*}(D(E), S(E))$. We have

$$
\begin{aligned}
\theta \circ \phi(x) & =\theta \circ \phi(Q, F, J)=\theta\left(F^{-1}(X), F\left|F^{-1}(X), J\right| F^{-1}(X)\right) \\
& =\theta(M, f, J \mid M)=\left(D\left(f^{*} E\right), \bar{f}, \pi^{*} J \mid M\right) .
\end{aligned}
$$

Identify $D\left(f^{*} E\right)$ with a tubular neighborhood of $M$ in $Q$. Then $(Q \times I, F, J)$ can be interpreted as a bordism between $x=(Q, F, J)$ and $\left(D\left(f^{*} E\right), F \mid D\left(f^{*} E\right)\right.$, $J \mid D\left(f^{*} E\right)$ ) by smoothing the corner at $Q \times 1$ and introducing a comer at $S\left(f^{*} E\right) \times 1$. See, e.g., [1]. Since the maps $\bar{f}$ and $F:\left(D\left(f^{*} E\right), S\left(f^{*} E\right)\right) \rightarrow$ $(D(E), S(E))$ are homotopic by standard arguments, we have $\theta \circ \phi(x)=x$.

Example. Let $G$ be a compact Lie group and let ad: $G \rightarrow O(n)$ be the adjoint representation. Let $E \rightarrow B G$ be the vector bundle induced by $B$ ad: $B G \rightarrow B O(n)$. Then $\Omega_{*}^{U}(B G, E)=U_{*}(D(E), S(E))$.

Proposition 2. $\Omega_{*}^{U}(B G, E)$ may be identified with the bordism group of stably almost complex manifolds with free $G$ action preserving the stable almost complex structure.

Proof. Let $(M, f, J) \in \Omega_{*}^{U}(B G, E)$, and let $P \stackrel{\pi}{\rightarrow} M$ be the principle $G$ bundle over $M$ induced by $f$. Then $T(P) / G=T(M) \oplus T_{F} / G$, where $T_{F}$ is the tangent bundle along the fibre. However, $T_{F} / G$ is just $f^{*} E$ by [2]. Thus $J$ provides a stable almost complex structure on $P$ which is preserved by the $G$ action. Conversely, if $P$ has a stable almost complex structure preserved by the free action of $G, T(P) / G$ has such a structure.

Corollary. If $H_{*}(B G)$ is torsion free then $\Omega_{*}^{U}(B G, E)$ is a free $U_{*}$ module for any orientable bundle $E$. 
Proof. $\Omega_{*}^{U}(B G, E)=U_{*}(D(E), S(E))$ by Proposition 1. There is a spectral sequence with $E_{p, q}^{2}=H_{p}\left(D(E), S(E) ; U_{q}\right)$ which converges to $U_{*}(D(E), S(E))$. Since $H_{*}(B G)$ is torsion free, $H_{*}(B T)$ maps epimorphically to $H_{*}(B G)$; hence $H_{*}(B G)$ is nonzero only in even dimensions. By the Thom isomorphism for the orientable bundle $E, H_{p}(D(E), S(E))=H_{p-\operatorname{dim} E}(B G)$. Since $U_{q}=0$ for $q$ odd, $E_{p, q}^{2}=0$ unless $p-\operatorname{dim} E \equiv q \equiv 0(\bmod 2)$, and hence all differentials in the spectral sequence are zero and $\Omega_{*}^{U}(B G, E)=$ $H_{*}(B G) \otimes U_{*}$ is a free $U_{*}$ module.

\section{BIBLIOGRAPHY}

1. R. Stong, Notes on cobordism theory, Math. Notes, Princeton Univ. Press, Princeton, N. J.; Univ. of Tokyo Press, Tokyo, 1968. MR 40 \# 2108.

2. C. Lazraov and A. G. Wasserman, Complex actions of Lie groups, Mem. Amer. Math. Soc. No. 137 (1973).

DEPARTMENT OF MATHEMATICS, CITY UNIVERSITY OF NEW YORK, LEHMAN COLLEGE, BRONX, NEW YORK 10468

DEPARTMENT OF MATHEMATICS, UNIVERSITY OF MICHIGAN, ANN ARBOR, MICHIGAN 48104 (Current address of Arthur Wasserman)

Current address (Connor Lazarov): School of Mathematics, Institute for Advanced Studies, Princeton, New Jersey 08540 